\title{
DIRECT PRODUCTS AND SUMS OF TORSION-FREE ABELIAN GROUPS
}

\author{
C. E. MURLEY
}

\begin{abstract}
Let $A$ be a finite rank, indecomposable torsion-free Abelian group whose $p$-ranks are less than two for all primes $p$. Let $G$ be a direct product of copies of $A$, and $B$ be a nonzero countable pure subgroup of $G$ such that $B$ is the span of the homomorphic images of $A$ in $B$. Then it is shown that $B$ is a direct sum of copies of $A$. This result is applied to obtain a Krull-Schmidt theorem for direct sums of groups $A$ from a semirigid class of groups. In particular, if the groups $A$ have rank one, then the well-known BaerKulikov-Kaplansky theorem is obtained.
\end{abstract}

All groups in this paper are torsion-free Abelian groups. Let $A$ be a group. Then the p-rank of $A, r_{p}(A)$, is the $Z / p Z$-dimension of $A / p A$ for $p$ a rational prime, $r(A)$ denotes the rank of $A$ and $A$ is called a $J$-group if every subgroup of finite index is isomorphic to $A$. Let $\mathscr{E}$ denote the class of indecomposable groups $A$ of finite rank such that $r_{p}(A) \leqq 1$ for all primes $p$. For general information about the class $\mathscr{E}$, the reader is referred to $\S \S 4$ and 5 of [10] where a slightly larger class of groups is studied. As in [2], a subfunctor of the identity $S(-)$ on $\mathscr{A}$, the category of $Z$-modules, is called a socle if $S^{2}=S$. Note that socles commute with direct sums. Let $X$ be a set of groups and $G \in \mathrm{ob}(\mathscr{A})$. Then $S_{X}(G)=\sum \phi(A)$ where $\phi$ ranges over $\operatorname{Hom}(A, G)$ and $A$ over $X$ defines a socle. We call $S_{X}(-)$ the socle associated with $X$. For all unexplained terminology, the reader is referred to [5].

\section{The homogeneous case.}

LEMMA 1. $A \in \mathscr{E}$ if and only if $A$ is a finite rank J-group such that every endomorphism is an integral multiple of an automorphism.

Proof. This is an easy consequence of Theorems 2 and 4 in [10].

Lemma 2. Let $A \in \mathscr{E}, G=\prod_{i \in I} A_{i}$ where $A_{i} \cong A$ for all $i$ and $D$ be $a$ pure subgroup of $G$ where $D \cong A$. Then $D$ is a summand of $G$.

Received by the editors April 2, 1972 and, in revised form, July 24, 1972.

AMS (MOS) subject classifications (1970). Primary 20K25, 20K15; Secondary $20 \mathrm{~K} 20$.

Key words and phrases. p-rank of a group, $J$-group, strongly homogeneous group, semirigid class of groups, cohesive group.

(C) American Mathematical Society 1973 
Proof. We let $\pi_{i}$ denote the projection of $G$ onto $A_{i}$ and identify $A_{i}$ with its natural injection in $G$. Let $i$ be an index such that $\pi_{i}(D) \neq 0, \pi_{i}^{\prime}$ denote the restriction of $\pi_{i}$ to $D$ and $\lambda_{i}$ be an isomorphism on $A_{i}$ onto $D$. Then $\lambda_{i} \pi_{i}^{\prime}$ is a nonzero endomorphism of $D$ and so by Lemma $1, \lambda_{i} \pi_{i}^{\prime}=$ $n \theta_{i}$ for some automorphism $\theta_{i}$ of $D$ and $n>0$. Let $\phi_{n}=\theta_{i}^{-1} \lambda_{i} \pi_{i}$. Then $\phi_{n}$ restricted to $D$ is just multiplication by the integer $n$. We may assume that if $p A=A$, then $p \nmid n$. Let $T$ be the set of prime divisors of $n$ and for $x \in G$, let $H_{p}^{G}(x)$ denote the $p$-height of $x$ in $G$. For each $p \in T$, there is an $x_{p} \in D$ such that $H_{p}^{G}\left(x_{p}\right)=0$ by the purity of $D$ in $G$. Let $x_{p}=\left\langle a_{j}\right\rangle_{j \in I}$ where $a_{j} \in A_{j}$. Then it follows that there is an index $j \neq i$ such that $H_{p}^{G}\left(a_{j}\right)=0$. If $\lambda_{j}$ is an isomorphism on $A_{j}$ onto $D$, then again by Lemma $1, \lambda_{j} \pi_{j}^{\prime}=$ $n_{p} \theta_{j}$ for some automorphism $\theta_{j}$ of $D$ and $n_{p}>0$. Let $\phi_{p}=\theta_{j}^{-1} \lambda_{j} \pi_{j}$. Then $\phi_{p}$ restricted to $D$ is multiplication by $n_{p}$. Since $H_{p}^{G}\left(\phi_{p}\left(x_{p}\right)\right)=H_{p}^{G}\left(\pi_{j}\left(x_{p}\right)\right)=$ $H_{p}^{G}\left(a_{j}\right)=0, p \nmid n_{p}$. Thus, $\{n\} \cup\left\{n_{p}\right\}_{p \in T}$ has a g.c.d. of 1 and so $n m+$ $\sum_{p \in T} n_{p} m_{p}=1$ for some integers $m, m_{p}$. Let $\phi=\sum_{p \in T} m_{p} \phi_{p}+m \phi_{n}$. Then $\phi$ is a homomorphism on $G$ into $D$ such that $\phi$ restricted to $D$ is the identity map. Hence, $D$ is a summand of $G$.

THEOREM 1. Let $A \in \mathscr{E}$ and $S(-)$ be the socle associated with $\{A\}$. Let $G=\prod_{i \in I} A_{i}$ where $A \cong A_{i}$ for all $i$ and $B$ be a countable pure nonzero subgroup of $G$. Then $B$ is a direct sum of copies of $A$ whenever $S(B)=B$.

Proof. We decompose the proof into three steps; we let $\pi_{i}$ denote the projection of $G$ onto $A_{i}$ and identify $A_{i}$ with its natural injection in $G$.

(i) If $0 \neq \phi \in \operatorname{Hom}(A, G)$, then there is a $\lambda \in \operatorname{Hom}(A, G)$ such that $\lambda(A) \cong A$ and $\lambda(A)=\mathrm{PH}(\phi(A))$, the pure hull of $\phi(A)$ in $G$. To prove this, let $i$ be an index such that $\pi_{i} \phi \neq 0, \lambda_{i}$ be an isomorphism on $A_{i}$ onto $A$ and $\theta=\lambda_{i} \pi_{i} \phi$. Then $\theta(A)=n A$ for some $n>0$ by Lemma 1 . It follows that $\phi$ is monic and since $r(A)<\infty, \lambda_{i} \pi_{i}$ is monic on $\phi(A)$. Since $\phi(A)$ is an essential subgroup of $\mathrm{PH}(\phi(A)), \lambda_{i} \pi_{i}$ is monic on $\mathrm{PH}(\phi(A))$. Since $A$ is a $J$-group [Lemma 1] and $n A \subseteq \lambda_{i} \pi_{i}(\mathrm{PH}(\phi(A))) \subseteq A, \lambda_{i} \pi_{i}(\mathrm{PH}(\phi(A))) \cong A$ and so $\phi(A) \cong \mathrm{PH}(\phi(A))$. Let $\rho$ be an isomorphism on $\phi(A)$ onto $\mathrm{PH}(\phi(A))$. Then $\lambda=\rho \phi$ is the desired map.

(ii) Any element of $B$ is contained in a summand of $B$ which is a finite direct sum of copies of $A$. To prove this, note that since $S(B)=B$, for each $x$ in $B$, there is a finite subset $T_{x}$ of $\operatorname{Hom}(A, B) \backslash\{0\}$ such that $x \in$ $\sum_{\phi \in T_{x}} \phi(A)$. In view of (i) and the purity of $B$ in $G$, we may assume that for $\phi \in T_{x}, \phi(A)$ is a pure copy of $A$ in $B$. Let $\lambda \in T_{x}$. Then $\lambda(A)$ is a summand of $G$ by Lemma 2 and so a summand of $B$. Let $B=\lambda(A) \oplus C$ and $x=y+z$ for $y \in \lambda(A), z \in C$. If $\operatorname{card}\left(T_{x}\right)=1$, then we are done. Assume (ii) is true for all $x$ in $B$ which have a $T_{x} \subseteq \operatorname{Hom}(A, B)$ with $\operatorname{card}\left(T_{x}\right) \leqq n$. Suppose $\operatorname{card}\left(T_{x}\right)=n+.1$. Let $\pi$ be the projection on $B$ onto $C$. Then $z \in \sum \pi \phi(A)$ where $\phi$ ranges over $T_{x} \backslash\{\lambda\}$. It follows from our assumption 
that $z$ is contained in a summand of $C$ that is a finite direct sum of copies of $A$, and, therefore, $x$ is contained in a summand of $B$ that is a finite direct sum of copies of $A$.

(iii) To complete the proof of the theorem, we proceed as in $[8$, Theorem 2]. Let $\lambda$ be an ordinal such that $\lambda \leqq \omega$, the first limit ordinal, and $X=$ $\left\{x_{i}\right\}_{i<\lambda}$ be a maximal independent set of $B$. In view of (ii), $B=B_{1} \oplus C_{1}$ where $x_{1} \in B_{1}$, which is a finite direct sum of copies of $A$. Let $y_{2}$ be the projection of $x_{2}$ on $C_{1}$. Then again by (ii), $B=B_{1} \oplus B_{2} \oplus C_{2}$ where $y_{2} \in B_{2}$, which is a finite direct sum of copies of $A$. Continuing in this way we obtain a pure subgroup, $\bigoplus_{i<\lambda} B_{i}$, of $B$ which contains $X$. Hence, $B=$ $\oplus_{i<\lambda} B_{i}$, which completes the proof.

Corollary 1. Let $A \in \mathscr{E}, G=\bigoplus_{i \in I} A_{i}$ where $A \cong A_{i}$ and $S(-)$ be the socle associated with $\{A\}$. Then any countable nonzero pure subgroup $B$ of $G$ such that $S(B)=B$ is a direct sum of copies of $A$ and any nonzero summand of $G$ is a direct sum of copies of $A$.

Proof. The first part follows by observing that $G$ is pure in $\prod_{i \in I} A_{i}$ and applying Theorem 1. For the second part, let $G=B \oplus C$. Then in view of Kaplansky [7], we may assume that $B$ is countable. Now $G=$ $\bigoplus_{i \in I} S\left(A_{i}\right)=S(G)=S(B) \oplus S(C)$ where $S(-)$ is the socle associated with $\{A\}$. Thus, $B=S(B)$ and the result follows from the first part.

The countability hypothesis in Theorem 1 is a necessary condition as may be seen by considering the Specker group, i.e. a countably infinite product of copies of $Z$. On the other hand, D. Arnold has informed me (unpublished) that the countability hypothesis in Corollary 1 is unnecessary. This is easy to see when the group $A$ in Corollary 1 is strongly homogeneous. Although this is a special case of $\mathrm{D}$. Arnold's result, it seems worthwhile to make this short proof available. Recall that a group $A$ is strongly homogeneous [11] if given two rank one, pure subgroups of $A$, there is an automorphism of $A$ which induces an isomorphism between these two groups. The structure of the strongly homogeneous groups in $\mathscr{E}$ is known, in view of [11, Theorem 4] and [10, Theorem 5].

THEOREM 2. Let $A$ be a strongly homogeneous group in $\mathscr{E}, S(-)$ be the socle associated with $\{A\}$ and $G$ be a direct sum of copies of $A$. Then a pure subgroup $B$ of $G$ is a direct sum of copies of $A$ whenever $S(B)=B \neq 0$.

Proof. We may assume that $A$ is reduced. Let $R$ be the endomorphism ring of some reduced group in $\mathscr{E}$. Then $R$ is a Principal Ideal Domain [P.I.D.] and $Z$ is dense in $R$ with respect to the $Z$-adic topology (see [10, Corollary 7]). The denseness of $Z$ in $R$ implies that a reduced $Z$ module is a (unitary) $R$-module in at most one way and that given two $R$ modules $M$ and $N$ which are reduced as $Z$-modules, the $R$-homomorphisms 
and $Z$-homomorphisms of $M$ into $N$ coincide. In addition, suppose that $N$ is a torsion-free $R$-module and $M$ is an $R$-submodule. Then $M$ is a pure $R$-submodule of $N$ whenever $M$ is a pure subgroup of $N$ (since every element of $R$ is an associate of an integer by Lemma 1). Now a necessary and sufficient condition that a group in $\mathscr{E}$ be strongly homogeneous is that it be a rank one, torsion-free module over its endomorphism ring (see [10, Theorem 5]). Hence, if $R=\operatorname{End}(A)$, then $G$ is a torsion-free $R$-module which is a direct sum of isomorphic rank one $R$-submodules, i.e. $G$ is a homogeneous, completely decomposable $R$-module. The condition that $S(B)=B$ implies that $B$ is a sum of $R$-submodules of $G$ and so $B$ is an $R$-submodule of $G$. Since $B$ is a pure subgroup of $G, B$ is a pure $R$-submodule of $G$. The proof is completed by applying the wellknown theorem of Baer [1], i.e. pure submodules of homogeneous, completely decomposable $R$-modules are completely decomposable, to $B$. Here, of course, we need that $R$ is a P.I.D.

Since a pure subgroup of a group in $\mathscr{E}$ is a direct sum of groups in $\mathscr{E}$, one might expect a pure subgroup of $G$, which is as in Corollary 1 , to be a direct sum of groups in $\mathscr{E}$. We give an example of a group $G=A \oplus A \oplus A$ for some $A \in \mathscr{E}$ which has a pure indecomposable subgroup $B$ not in $\mathscr{E}$ : Let $p_{1}, p_{2}, p_{3}$ be distinct primes and $A \in \mathscr{E}$ such that $r(A)=3, r\left(p_{i}^{\omega} A\right)=2$ for $i=1,2,3, r\left(p_{i}^{\omega} A \cap p_{j}^{\omega} A\right)=1$ for $i \neq j, \bigcap_{i=1}^{3} p_{i}^{\omega} A=\{0\}$, and $p^{\omega} A=\{0\}$ for $p \neq p_{i}$. Such a group $A$ exists by the construction in Example 2 [10]. Let $\dot{G}=A_{1} \oplus A_{2} \oplus A_{3}$ where $A_{i} \cong A$ and $0 \neq a_{1} \in p_{1}^{\omega} A_{1} \cap p_{2}^{\omega} A_{1}, \quad 0 \neq a_{2} \in p_{1}^{\omega} A_{2} \cap$ $p_{3}^{\omega} A_{2}, \quad 0 \neq a_{3} \in p_{2}^{\omega} A_{3} \cap p_{3}^{\omega} A_{3}$. Now let $C=\oplus_{i=1}^{3} \operatorname{PH}^{G}\left(a_{i}\right)$ where $\mathrm{PH}^{G}\left(a_{i}\right)$ denotes the pure hull of $\left(a_{i}\right)$ in $G$. Then $C$ contains an indecomposable pure subgroup $B$ of rank 2 , e.g. take $B=\mathrm{PH}^{C}\left(b_{1}, b_{2}\right)$ where $b_{1}=a_{1}+a_{2}$, $b_{2}=a_{2}+a_{3}$ and show that $B$ is indecomposable as in Erdös' example [4, p. 166]. Since $r_{p}(C)=3$ for $p \neq p_{i}$ and $r_{p}(C / B) \leqq 1, r_{p}(B) \geqq 2$ for $p \neq p_{i}$, i.e. $B \notin \mathscr{E}$.

2. Semirigid subclasses of $\mathscr{E}$. We call, as in Charles [2], a class of groups $\left\{A_{i}\right\}_{i \in I}$ semirigid if $I$ can be partially ordered such that for $i, j \in I$, $i \leqq j$ if and only if $\operatorname{Hom}\left(A_{i}, A_{j}\right) \neq 0$. Let $\mathscr{F}=\left\{A_{i}\right\}_{i \in I}$ be a semirigid class and $G$ be a direct sum of groups, each isomorphic to some group in $\mathscr{F}$. Then $G=\bigoplus_{i \in I} G(i)$ where $G(i)$ is either the zero group or a direct sum of copies of $A_{i}$. We call $G(i)$ an $A_{i}$-homogeneous component of $G$. If $S_{i}(-)$ and $S_{i}^{*}(-)$ are the socles associated with $\left\{A_{j} \in \mathscr{F} \mid j \geqq i\right\}$ and $\left\{A_{j} \in \mathscr{F} \mid j>i\right\}$ respectively, then it is easily checked that $S_{i}(G) / S_{i}^{*}(G) \cong$ $G(i)$. Thus, an $A_{i}$-homogeneous component of $G$ is unique up to isomorphism. A modest argument, which uses Kaplansky [7] and involves computations with the socles $S_{i}(-)$ and $S_{i}^{*}(-)$, gives the following special version of Charles [2, Theorem 2.13]: Let $\mathscr{F}=\left\{A_{i}\right\}_{i \in I}$ be a semirigid class of countable groups, $G$ be a direct sum of groups, each isomorphic 
to some group in $\mathscr{F}$, and $G=\bigoplus_{i \in I} G(i)$. Then for any summand $B$ of $G$, $B=\bigoplus_{i \in I} B(i)$ where $B(i)$ is isomorphic to a summand of $G(i)$.

THEOREM 3. Let $\mathscr{F}$ be a semirigid subclass of $\mathscr{E}$ and $G=\bigoplus_{i \in I} A_{i}$ where each $A_{i}$ is isomorphic to some group in $\mathscr{F}$. Then any direct sum decomposition of $G$ refines to a decomposition isomorphic the given decomposition. Equivalently, any nonzero summand of $G$ is a direct sum of groups, each isomorphic to one of the original summands $A_{i}$.

Proof. Since the $A$-homogeneous components of $G$ are isomorphic for a fixed $A$ in $\mathscr{F}$, the theorem is immediate from the above version of Charles' theorem and Corollary 1.

Although $\mathscr{E}$ has abundant semirigid subclasses, it is easy to see that $\mathscr{E}$ is not itself a semirigid class. On the other hand, for $\mathscr{F} \subseteq \mathscr{E}$, it is not clear that the semirigidity of $\mathscr{F}$ is necessary for Theorem 3 to hold. In fact, if the hypotheses of Theorem 3 are suitably altered, then it should be possible to obtain a theorem similar to ours without requiring $\mathscr{F}$ to be semirigid. For example, let $\mathscr{F}=\{A, B\} \subset \mathscr{E}$ such that $A \not \Delta B, \operatorname{Hom}(A, B) \neq 0$ and $\operatorname{Hom}(B, A) \neq 0$ (the existence of such a pair of groups will be clear from a later example). Then $\mathscr{F}$ is not semirigid and since $A$ and $B$ are indecomposable $J$-groups, $A$ and $B$ are strongly indecomposable groups, i.e. subgroups of finite index are indecomposable. It follows from Jónsson [6] that the Krull-Schmidt theorem holds for $G=A \oplus B$.

In the remainder of this section we consider some semirigid subclasses of $\mathscr{E}$ which appear to be of interest. Since a semirigid class cannot, as defined, contain two distinct isomorphic groups, we will always identify the isomorphic groups in any given class of groups. Let $\mathrm{Zp}$ denote the local subring of the rationals $Q$ determined by the prime $p$ and $Z p^{*}$ denote the ring of $p$-adic integers. Recall that for a group $A, r_{p}(A)=1$ and $p^{\omega} A=0$ if and only if $Z p \otimes A$ is a pure subgroup of $Z p^{*}$. Such groups are precisely the $p$-pure subgroups of $Z p^{*}$, which are necessarily indecomposable (since the pure subgroups of $Z p^{*}$ are indecomposable).

Definition. $\mathscr{F} p=\left\{A \in \mathscr{E} \mid p^{\omega} A=0\right\}$ for a fixed prime $p$ and let $\mathscr{C}$ be the class of finite rank, indecomposable groups $A$ such that the nonzero homomorphisms on $A$ into reduced groups are monic. The groups in $\mathscr{C}$ are called cohesive groups [3].

LEMMA 3. $\mathscr{C} \cup \mathscr{F} p$ is a semirigid subclass of $\mathscr{E}$ such that $\mathscr{C} \backslash \mathscr{F} p$ and $\mathscr{F} p \backslash \mathscr{C}$ are uncountable sets.

Proof. It is well known that $\mathscr{C}=\left\{A \in \mathscr{E} \mid p A \neq A\right.$ implies $\left.p^{\omega} A=0\right\}$ (see [3]) and it is immediate from [10, Example 2] that the complements are uncountable. Let $A, B \in \mathscr{F} p$ and $0 \neq \phi \in \operatorname{Hom}(A, B)$. Then $0 \neq \mathrm{id} \otimes \phi$ : $Z p \otimes A \rightarrow Z p \otimes B$ is monic, since it is multiplication by a nonzero $p$-adic 
integer, and so $\phi$ is monic. Since the groups in $\mathscr{E}$ are $J$-groups, it follows that $\mathscr{C}$ and $\mathscr{F} p$ are semirigid subclasses of $\mathscr{E}$. On the other hand, if $A \in \mathscr{C} \mid \mathscr{F} p$, then $p A=A$ and so $\operatorname{Hom}(A, B)=0$ for $B \in \mathscr{F} p$. It follows that $\mathscr{C} \cup \mathscr{F} p$ is semirigid.

Corollary 2. If $G=\bigoplus_{i \in I} A_{i}$ where $A_{i} \in \mathscr{C} \cup \mathscr{F} p$, then any direct sum decomposition of $G$ refines to the given decomposition and any nonzero summand of $G$ is a direct sum of subgroups isomorphic to the $A_{i}$.

REMARK. Since the rank one groups are cohesive, a special case of Corollary 2 is the Baer-Kulikov-Kaplansky theorem, i.e. direct summands of completely decomposable groups are completely decomposable (see [1], [9], [7]). In addition, Proposition 4 in [12] is the special case of Corollary 2 where the summands $A_{i}$ are from the class of finite rank, pure subgroups of $Z p^{*}$ ( $p$ fixed), which we symbolically denote by $Z p \otimes \mathscr{F} p$. It follows from [12, Proposition 1], [11, Theorem 4] and [10, Corollary 9] that a reduced group $A$ in $\mathscr{E}$ has the (finite) exchange property (see [12]) if and only if $A \in Z p \otimes \mathscr{F} p$ for some prime $p$. R. B. Warfield has given in [13] a Krull-Schmidt theorem for direct sums of arbitrary Abelian groups which, in particular, have the finite exchange property. Therefore, Theorem 3 coincides with [13, Theorem 2] only in the case where the semirigid class $\mathscr{F}$ in Theorem 3 is a subclass of $\{Q\} \cup\{Z p \otimes \mathscr{F} p\}, p \in$ primes. Finally, we note another special case of Corollary 2 by observing that $\mathscr{C}$ contains the strongly homogeneous groups in $\mathscr{E}$.

Let $n>0$ and $\mathscr{E}_{n}$ denote the class of rank $n$ groups in $\mathscr{E}$, e.g. $\mathscr{E}_{1}$ is precisely the class of rank one groups. Then $\mathscr{E}_{n}$ is semirigid if and only if $n=1$. To see this, let $n>1$ and we exhibit two groups $A$ and $B$ in $\mathscr{E}_{n}$ such that $\operatorname{Hom}(A, B) \neq 0$ and $\operatorname{Hom}(B, A) \neq 0$ but $A \not B$ :

Let $p, q$ be distinct primes, $A \in \mathscr{F} p \cap \mathscr{E}_{n}, B \in \mathscr{F} q \cap \mathscr{E}_{n}$ such that $r\left(q^{\omega} A\right)=r\left(p^{\omega} B\right)=n-1$ and $A, B$ are divisible by all other primes. Such groups are easy to construct (see [10, Example 2]) and clearly $A \not B$. Since $A / q^{\omega} A \cong Z q$ and $B / p^{\omega} B \cong Z p, A / q^{\omega} A \rightarrow B$ and $B / p^{\omega} B \rightarrow A$.

In particular, this example shows that for $n>1$ and $p \neq q,(\mathscr{F} p \cup \mathscr{F} q) \cap \mathscr{E}_{n}$ is not semirigid. Since the set of all semirigid subclasses of $\mathscr{E}_{n}$ (with inclusion as a P.O.) is inductive, every semirigid subclass of $\mathscr{E}_{n}$ is contained in a maximal semirigid [m.s.r.] subclass of $\mathscr{E}_{n}$. Thus, for each prime $p$, $\mathscr{F} p \cap \mathscr{E}_{n}$ is contained in an m.s.r. subclass of $\mathscr{E}_{n}$ and so for $n>0$, in view of the above example, there are an infinite number of distinct m.s.r. subclasses of $\mathscr{E}_{n}$. Now $\mathscr{C} \cap \mathscr{E}_{n}$ is uncountable (see [3] or [10]) and it is easy to see that $\mathscr{C} \cap \mathscr{E}_{n}$ is contained in every m.s.r. subclass of $\mathscr{E}_{n}$. Thus, every m.s.r. subclass of $\mathscr{E}_{n}$ is uncountable. Although we are unable to identify the m.s.r. subclasses of $\mathscr{E}_{n}$, we note in the following lemma what appears to be a fairly large semirigid subclass of $\mathscr{E}_{n}$. 
LEMMA 4. Let $n>2$ and $\mathscr{F}=\left\{A \in \mathscr{E}_{n} \mid p A \neq A\right.$ implies $\left.r\left(p^{\omega} A\right)<[n / 2]\right\}$. Then $\mathscr{F}$ is a semirigid class where $\mathscr{F} \backslash(\mathscr{C} \cup \mathscr{F} p)$ is an uncountable set.

Proof. That the complement is uncountable is immediate from [10, Example 2]. For $A, B \in \mathscr{F}$ and $0 \neq \phi \in \operatorname{Hom}(A, B)$, it is a modest computation, which uses the relation $r_{p}(A)=r_{p}(\operatorname{ker} \phi)+r_{p}(\phi(A))$, to show $\phi$ is monic. Hence, $\mathscr{F}$ is semirigid.

\section{REFERENCES}

1. R. Baer, Abelian groups without elements of infinite order, Duke Math. J. 3 (1937), 68-122.

2. B. Charles, Sous-groupes fonctoriels et topologies, Studies on Abelian Groups (Sympos., Montpellier, 1967), Dunod, Paris; Springer, Berlin, 1968, pp. 75-92. MR 39 \#1547.

3. D. W. Dubois, Cohesive groups and p-adic integers, Publ. Math. Debrecen 12 (1965), 51-58. MR 36 \#3875.

4. L. Fuchs, Abelian groups, Publ. House Hungarian Acad. Sci., Budapest, 1958. MR 21 \#5672.

5. —_, Infinite abelian groups. Vol. I, Pure and Appl. Math., vol. 36, Academic Press, New York, 1970. MR 41 \#333.

6. B. Jónsson, On direct decompositions of torsion free Abelian groups, Math. Scand. 7 (1959), 361-371. MR 23 \#A204.

7. I. Kaplansky, Projective modules, Ann. of Math. (2) 68 (1958), 372-377. MR 20 \#6453.

8. G. Kolletis, Homogeneously decomposable modules, Studies on Abelian Groups, Dunod, Paris, 1968, pp. 223-238.

9. L. Ja. Kulikov, On direct decompositions of groups, Ukrain. Mat. Ž. 4 (1952), 347-372; English transl., Amer. Math. Soc. Transl. (2) 2 (1956), 23-87. MR 15, 396; MR 17, 824.

10. C. E. Murley, The classification of certain classes of torsion free Abelian groups, Pacific J. Math. 40 (1972), 647-665.

11. F. Richman, $A$ class of rank-2 torsion free groups, Studies in Abelian Groups (Sympos., Montpellier, 1967), Dunod, Paris; Springer, Berlin, 1968, pp. 327-333. MR 39 \#5695.

12. R. B. Warfield, Jr., A Krull-Schmidt theorem for infinite sums of modules, Proc. Amer. Math. Soc. 22 (1969), 460-465. MR 39 \#4213.

13. _ An isomorphism refinement theorem for Abelian groups, Pacific J. Math. 34 (1970), 237-255. MR 42 \#1896.

Department of Mathematics, University of Victoria, Victoria, B.C., Canada 